\title{
CARACTERIZAÇÃO E AVALIAÇÃO DE TRÊS GRUPOS DE ARROZ-DE-SEQUEIRO DE DIFERENTES PROCEDÊNCIAS POR MEIO DA SENSITIVIDADE À RADIAÇÃO GAMA ${ }^{(1)}$
}

\author{
LUÍS ROBERTO FRANCO RODRIGUES ${ }^{(2)}$; AKIHIKO ANDO(3)
}

\begin{abstract}
RESUMO
A caracterização e avaliação de variedades de arroz auxiliam na seleção dos parentais e na ampliação da base genética dos programas de melhoramento. Este trabalho teve como objetivo caracterizar e avaliar três grupos de arroz-de-sequeiro (Oryza sativa L.) de diferentes procedências por meio da sensitividade à radiação gama. Sementes de 84 variedades procedentes do Japão, Filipinas e Brasil foram submetidas a várias dosagens de radiação gama e semeadas em caixas de madeira no delineamento em blocos completos ao acaso com três repetições. O experimento foi instalado em casa de vegetação, em 1991, no Centro de Energia Nuclear na Agricultura (CENA), da Universidade de São Paulo (USP). Mediu-se a sensitividade por meio dos efeitos fisiológicos produzidos na geração $\mathrm{M}_{1}$; determinaram-se as porcentagens de emergência, a sobrevivência e a altura das plântulas. Os resultados mostraram diferenças significativas de sensitividade à radiação gama entre grupos em todos os caracteres avaliados.

Palavras-chave: arroz-de-sequeiro, Oryza sativa L., Índica, Japônica, emergência, altura de
\end{abstract} plântula, taxa de sobrevivência, sensitividade, radiação gama.

\section{ABSTRACT \\ CHARACTERIZATION AND EVALUATION OF THREE GROUPS OF RICE UPLAND OF DIFFERENT ORIGINS THROUGH GAMMA-RAY SENSITIVITY}

The characterization and evaluation of varieties of rice aid in the parents selection and in the increase of the genetic base of breeding programs. The present work was carried out to characterize and to evaluate three groups of rice upland (Oryza sativa L.) of different origins through gamma-ray sensitivity. Seeds of 84 varieties collected from Japan, Philippines and Brazil, were submitted to various doses of gammaradiation and sown in wooden boxes. The experiment was conducted in 1991 at greenhouse according to randomized complete block design with three replications. Physiological effects caused by radiation in the $M_{1}$ generation, such as seed emergency, seedling height and survival rate, were determined and evaluated. The results showed significant differences of sensitivity to the radiation among three groups for all traits evaluated.

Key words: upland rice, Oryza sativa L., Indica, Japônica, seed emergency, seedling height, survival rate, sensitivity, gamma-radiation.

\section{INTRODUÇÃO}

O arroz (Oryza sativa L.) é uma das culturas principais mais importantes, pois alimenta mais da metade da população mundial e fornece vários subprodutos.

Nos últimos anos, a variabilidade genética reduzida em função da base genética estreita, associada a práticas culturais modernas e aos cultivos sucessivos, tem aumentado a vulnerabilidade genética da cultura, principalmente à incidência de pragas e doenças
(KHush, 1991), bem como dificultado a superação de patamares de produtividade (GUIMARĀES et al., 1996; MontAlVÁn et al., 1998).

A caracterização e a avaliação de coleçôes de germoplasma têm auxiliado na identificação, na conservação e na maior exploração da variabilidade genética.

Os métodos bioquímicos tais como reação ao fenol, resistência ao clorato de potássio, teste do álcali e padrão isoenzimático, além de outros, têm sido muito úteis na

\footnotetext{
( $\left.{ }^{1}\right)$ Parte da dissertação de mestrado do primeiro autor apresentada à Escola Superior de Agricultura "Luiz de Queiroz", Universidade de São Paulo (ESALQ/USP). Recebido para publicação em 23 de outubro de 2000 e aceito em 28 de fevereiro de 2002.

$\left(^{2}\right)$ Departamento de Genética da Universidade Estadual Paulista (UNESP), Distrito de Rubião Júnior s/n, $18618-000$ Botucatu (SP).

$\left({ }^{3}\right)$ Centro de Energia Nuclear na Agricultura (CENA), Caixa Postal 96, 13400-970 Piracicaba (SP).
} 
caracterização e classificação das variedades de arroz em grupos Índica, Japônica Temperado e Japônica Tropical (KATO et al. 1928; OKA, 1958), para selecionar os parentais e ampliar a base genética dos programas de melhoramento (FERREIRA et al., 2000). Entretanto, esses métodos apresentam desvantagem porque inutilizam a amostra biológica. Desse modo, seria interessante o desenvolvimento de métodos que não inutilizassem o material a ser caracterizado e avaliado.

Desde os estudos iniciais feitos por JoHNSON (1933) e SMITH (1942), constata-se que variedades, linhagens, híbridos, grupos varietais, tecidos e órgãos vegetais e estádio fenológico da planta podem apresentar diferenças de sensitividade à radiação gama ou de radiossensitividade. Em milho, verificou-se que as linhagens são mais radiossensitivas que os híbridos simples e estes, por sua vez, mais radiossensitivos que os duplos (SARIC, 1961). Entre os grupos Índica e Japônica também há diferenças de radiossensitividade (FUJII, 1962).

O presente trabalho teve como objetivo caracterizar e avaliar três grupos de arroz-de-sequeiro procedentes do Japão, Filipinas e Brasil por meio da sensitividade à radiação gama.

\section{MATERIAL E MÉTODOS}

O Departamento de Genética da ESALQ/USP mantém uma coleção de germoplasma de arroz-desequeiro (Oryza sativa L.) procedente da Estação Experimental de Arroz-de-sequeiro do Japão, do International Rice Research Institute (IRRI) das Filipinas, do Instituto Agronômico (IAC), Campinas (SP) e do Centro Nacional de Pesquisa de Arroz e Feijão CNPAF/EMBRAPA, Goiânia (GO). Em 1990, a coleção foi renovada no campo experimental do próprio departamento. No período de março a abril, as sementes de cada variedade foram coletadas separadamente, pesadas e armazenadas em condições ambientais. Selecionaram-se, com base na maior produção de grãos, 84 variedades de procedências japonesa, filipina e brasileira mais bem adaptadas às condições climáticas de Piracicaba (SP) - Quadro 1. As variedades japonesas utilizadas pertencem ao grupo Japônica e as filipinas, ao grupo Índica. No grupo brasileiro foram identificadas, por meio de marcadores RAPD específicos, algumas variedades como pertencentes ao grupo Japônica, tais como Bico Ganga, Guaíra, Dourado Precoce, Pratão Precoce, Iguapé e Agulha ESAV (Ferreira et al., 2000).

Em dezembro de 1991, instalou-se o experimento em casa de vegetação do Centro de Energia Nuclear na Agricultura (CENA), da Universidade de São Paulo (USP). Amostras de 75 sementes secas, uniformes, férteis e com $14 \%$ de umidade foram submetidas à exposição aguda de radiação gama nas doses $0,60,120,180,240$, 300 e 360 Gy, utilizando-se como fonte de raios gama $\left({ }^{60} \mathrm{Co}\right)$ o irradiador Gammabeam 650 do CENA. A taxa de dose variou de um experimento para o outro, sendo a mínima de 1.070 Gy por hora e a máxima de 1.270 Gy por hora, mantendo-se constante a distância do material em relação à fonte.

Após a irradiação, as sementes irradiadas e as nãoirradiadas (controle) foram semeadas imediatamente em caixas de madeira de $1,10 \mathrm{~m} \mathrm{x} \mathrm{1,26} \mathrm{m} \mathrm{com} \mathrm{terra}$ preparada; adotou-se o delineamento em blocos completos ao acaso com três repetiçôes e 25 sementes por parcela, totalizando $20,79 \mathrm{~m}^{2}$ de área útil.

Mediu-se a radiossensitividade de plântulas M1 aos 7, 10 e 30 dias anotando-se respectivamente: o número de plântulas emergentes com $5 \mathrm{~mm}$ ou mais de altura; a altura das plântulas - do solo até o ponto mais alto da folha com maior altura, e o número de plântulas sobreviventes.

Os dados experimentais foram obtidos de 18 plantas por parcela, selecionadas aleatoriamente, totalizando 31.752 plântulas avaliadas. As análises estatísticas foram feitas com os dados das porcentagens de emergência e sobrevivência transformados em arco seno $\sqrt{x / 100}$, e com os dados originais de altura. Efetuou-se a análise de todos os caracteres com base no seguinte modelo:

$$
\bar{Y} i j k=m+g_{i}+v_{(i) j}+d_{k}+g d_{i k}+v_{(i) j k}+e_{i j k}
$$

em que:

Yijk: é o valor observado referente à parcela que recebeu o germoplasma j, do grupo i com a dosagem k;

m: é a média geral;

$\mathrm{g}_{\mathrm{i}}$ : é efeito fixo de grupo $\mathrm{i},(\mathrm{i}=1,2,3)$;

$\mathrm{v}_{(\mathrm{i})}$ : é o efeito fixo do germoplasma $\mathrm{j}$ dentro do grupo $\mathrm{i}$ $(j=1, \ldots, j)$;

$\mathrm{d}_{\mathrm{k}}$ : é o efeito fixo da dose $\mathrm{k}(\mathrm{k}=1, \ldots, \mathrm{k})$;

$\mathrm{gd}_{\mathrm{ik}}$ : é o efeito fixo da interação do grupo i com a dose k; $\mathrm{vd}_{\text {(i)k }}$ : é o efeito fixo da interação do germoplasma j com a dose $\mathrm{k}$ no grupo $\mathrm{i}$;

$\mathrm{e}_{\mathrm{ijk}}$ : erro médio associado à observação $\overline{\mathrm{Y}}_{\mathrm{ijk}}$.

\section{RESULTADOS E DISCUSSÃO}

O resultado das análises da variância evidenciou diferenças significativas entre as médias de grupo, de variedades dentro de grupo, de doses, de interaçóes de grupo $\mathrm{x}$ dose e de variedades $\mathrm{x}$ dose dentro de grupo, ao nível de $1 \%$ pelo teste $\mathrm{F}$ para todos os caracteres avaliados (Quadro 2).

O valor de $\mathrm{F}$ significativo para a altura já era esperado, uma vez que entre variedades e entre grupos existe grande variação. 
Quadro 1. Variedades japonesas, filipinas e brasileiras utilizadas na caracterização e avaliação de grupos de arroz-de-sequeiro por meio da sensitividade à radiação gama

\begin{tabular}{|c|c|c|c|c|c|}
\hline Número & Nome & Banco de origem & Número & Nome & Banco de origem \\
\hline 01 & Senshou & Japão & 43 & Khao Phe & Filipinas \\
\hline 02 & Oohata & " & 44 & Khao Khay & “" \\
\hline 03 & Habiro & “ & 45 & Khao Noi & “ \\
\hline 04 & Iwate Hata Mochi & “ & 46 & Daw Pao & “ \\
\hline 05 & Kyuushuu & “ & 47 & Mah Nam Pui & “ \\
\hline 06 & Ooohata Mochi & “ & 48 & Pah Yan & “ \\
\hline 07 & Kinryuu & “ & 49 & Khao Khao & “ \\
\hline 08 & Nasukogane & “ & 50 & Graboon & “ \\
\hline 09 & Kurasawa & “ & 51 & Khao Seng & “ \\
\hline 10 & Yonoyuki Mochi & “ & 52 & Khao Mai Khai & “ \\
\hline 11 & Terenzu & “ & 53 & Wiang & “ \\
\hline 12 & Gaisen Mochi & “ & 54 & Betete & “ \\
\hline 13 & Owari Hata Mochi & “ & 55 & Ketan Cere & “ \\
\hline 14 & Nourin 5 & “ & 56 & Selibon Siangan & “ \\
\hline 15 & Hakamuri & “ & 57 & Bico Ganga & Brasil \\
\hline 16 & Esojima & “ & 58 & Guaíra & “ \\
\hline 17 & Nourin Mochi 17 & “ & 59 & Dourado Precoce & “ \\
\hline 18 & Col/Fukui/1965 & “ & 60 & Amarelão & “ \\
\hline 19 & Bansei Mochi & “ & 61 & Pratão Precoce & “ \\
\hline 20 & Tomoe Mochi & “ & 62 & IPSL 469 & “ \\
\hline 21 & Nourin 16 & “ & 63 & IPSL 2270 & “ \\
\hline 23 & Kahee & “ & 65 & Iguapão & “ \\
\hline 24 & Ishiwari Mochi & “ & 66 & Iguapé & “ \\
\hline 25 & Hakabou Aikoku & “ & 67 & Montanha Liso & “ \\
\hline 26 & Urasan & “ & 68 & Pérola de Campinas & “ \\
\hline 27 & Yakari & “ & 69 & Guapinha & “ \\
\hline 28 & Bansei Tarou & “ & 70 & Agulha ESAV & “ \\
\hline 29 & E 425 & Filipinas & 71 & Enche Tulha & “ \\
\hline 30 & Matopu Garatas & " & 72 & Mogi & “ \\
\hline 31 & Daja & “ & 73 & Dourado Liso & “ \\
\hline 32 & Ketek & “ & 74 & Jaguarí & “ \\
\hline 33 & Sono Atom & “ & 75 & Cateto Dourado & “ \\
\hline 34 & Rusip & “ & 76 & Dourado Seco & “ \\
\hline 35 & Lokan & “ & 77 & Matão Branco & “ \\
\hline 36 & Ketji & “ & 78 & Pratão de Campinas & “ \\
\hline 37 & Serung & “ & 79 & Pindorama & “ \\
\hline 38 & Menurun & “ & 80 & Dourado Agulha2 & “ \\
\hline 39 & Majangan & “ & 81 & Biriguí & “ \\
\hline 40 & Lembese & “ & 82 & $9-v-11$ & “ \\
\hline 41 & Tjempo Tsino & “ & 83 & $11-\mathrm{v}-22$ & “ \\
\hline 42 & Khao Sum neua2 & “ & 84 & $11-\mathrm{v}-26$ & “ \\
\hline
\end{tabular}

Quadro 2. Resumo das análises da variância da emergência, altura e sobrevivência de plântulas $M_{1}$ de 84 variedades de arroz-desequeiro irradiadas com diferentes doses de raios gama. Piracicaba (SP), 1991

\begin{tabular}{lrccc}
\hline Fontes de variação & G.L. & \multicolumn{3}{c}{ Quadrados médios } \\
\cline { 3 - 5 } & & Emergência & Altura & Sobrevivência \\
\hline Grupos & 2 & $3.719,57^{* *}$ & $17,71^{* *}$ & $3.688,09^{* *}$ \\
Variedades/grupos & 81 & $866,71^{* *}$ & $74,31^{* *}$ & $1.290,84^{* *}$ \\
Dose & 6 & $3.189,45^{* *}$ & $1.330,20^{* *}$ & $5.891,14^{* *}$ \\
Grupo x dose & 12 & $209,83^{* *}$ & $5,46^{* *}$ & $156,14^{* *}$ \\
Variedade x dose/grupo & 486 & $134,92^{* *}$ & $7,74^{* *}$ & $144,36^{* *}$ \\
Resíduo & 1.176 & 74,77 & 1,20 & 71,90 \\
\hline Média & & 67,29 & 10,98 & 65,15 \\
CV $(\%)$ & 12,85 & 9,98 & 13,01 \\
\hline
\end{tabular}

**: Significativo a $1 \%$. 
Geralmente, as variedades filipinas e brasileiras são mais altas que as japonesas. As diferenças encontradas em porcentagem de emergência podem estar relacionadas com o grau de dormência das sementes. No presente trabalho não foi medida a velocidade de germinação em períodos diversos; verificou-se, no entanto, em Roberts (1963), que variedades do grupo Japônica apresentaram menor grau de dormência que as variedades do grupo Índica. As diferenças observadas entre doses de radiação gama são comuns em experimentos em que se utilizam mutagênicos. De acordo com o objetivo deste trabalho, foram encontradas diferenças para as interações entre grupos $\mathrm{x}$ dose, comprovando que os grupos responderam distintamente em relação às dosagens do mutagênico, existindo, portanto, diferenças de radiossensitividade entre eles. Os fatores responsáveis pela diferença de radiossensitividade entre variedades e entre grupos podem ser físicos (tipo de radiação, dose, taxa de dose, exposição aguda e crônica), biológicos (conteúdo de DNA, volume nuclear e do cromossomo interfásico, nível de ploidia, heterozigose), químicos e ambientais (teor de umidade, temperatura, tempo de armazenagem, presença de oxigênio, hidrogênio e ausência ou baixa concentração de substância protetoras nas sementes ou tecidos vegetais).

Uma relação importante foi encontrada entre o teor de umidade e a radiossensitividade (CALDECOTT, 1955). O autor verificou que em sementes com $16 \%$ de teor de umidade, uma dose de $45 \mathrm{Kr}$ de raios $\mathrm{X}$ produziu $32 \%$ de sobrevivência. A mesma porcentagem foi observada em sementes com $4 \%$ de umidade aplicando-se $10 \mathrm{Kr}$. Neste trabalho, a porcentagem de umidade das sementes não pode ser considerada a responsável pela diferença de radiossensitividade entre as variedades e entre grupos em razão da sua uniformidade que foi de $14 \%$.

A comparação das médias dentro de grupos pelo teste de Tukey para todos os caracteres encontra-se nos quadros 3 , 4 e 5 .

No quadro 3, nota-se que o número de plântulas M1 emergidas não diferiu significativamente em relação ao controle para os grupos japonês, filipino e brasileiro, até as doses de $60 \mathrm{~Gy}, 180 \mathrm{~Gy}$ e $180 \mathrm{~Gy}$ respectivamente. A redução da emergência de plântulas $M 1$ pode ser atribuída a efeitos fisiológicos de origem cromossômica e extracromossômica (GAUL, 1977).

Avaliando-se a altura, observa-se que os grupos japonês, filipino e brasileiro, até as doses de 60 Gy, 120 Gy e 60 Gy, respectivamente, não diferiram significativamente em relação ao controle (Quadro 4). A redução da altura também é atribuída a fatores fisiológicos, fato constatado em vários trabalhos. Para esse caráter a GR 50 (dose que causa 50\% de redução de altura) foi alcançada na dose de $360 \mathrm{~Gy}$.
Quadro 3. Médias e porcentagens médias de emergência de plântulas $M_{1}$ dentro de grupos ${ }^{(1)}$ em função das doses de radiação gama aplicadas em variedades de arroz-desequeiro. Piracicaba (SP) 1991

\begin{tabular}{ccc}
\hline Dose/Gy & Emergência & Média $^{(2)}$ \\
\hline & Japonês & $\%$ \\
0 & 70,61 & $(100,00)$ \\
60 & $68,13 \mathrm{ab}$ & $(96,49)$ \\
120 & $64,67 \mathrm{bc}$ & $(91,59)$ \\
180 & $65,03 \mathrm{bc}$ & $(92,10)$ \\
240 & $62,55 \mathrm{bc}$ & $(88,59)$ \\
300 & $63,82 \mathrm{~cd}$ & $(90,38)$ \\
360 & $60,62 \mathrm{~d}$ & $(85,85)$ \\
\hline CV $(\%)$ & 10,26 & - \\
D.M.S. & 5,80 & - \\
\hline & Filipino & \\
0 & $74,61 \mathrm{a}$ & $(100,00)$ \\
60 & $72,63 \mathrm{ab}$ & $(97,35)$ \\
120 & $70,53 \mathrm{ab}$ & $(96,05)$ \\
180 & $71,66 \mathrm{abc}$ & $(94,53)$ \\
240 & $68,76 \mathrm{bcd}$ & $(92,16)$ \\
300 & $66,24 \mathrm{~cd}$ & $(88,78)$ \\
360 & $65,02 \mathrm{~d}$ & $(87,15)$ \\
\hline CV $(\%)$ & 11,30 & - \\
D.M.S. & 5,70 & - \\
\hline & Brasileiro & \\
60 & $71,70 \mathrm{a}$ & $(100,00)$ \\
120 & $71,89 \mathrm{a}$ & $(100,30)$ \\
180 & $69,97 \mathrm{ab}$ & $(97,59)$ \\
240 & $66,67 \mathrm{ab}$ & $(92,68)$ \\
300 & $66,43 \mathrm{bc}$ & $(89,00)$ \\
360 & $63,81 \mathrm{~cd}$ & - \\
\hline CV $(\%)$ & $61,10 \mathrm{~d}$ & \\
D.M.S & 13,30 & \\
\hline & 5,30 & \\
\hline & & \\
\hline & $28,22)$ \\
\hline
\end{tabular}

(1) Avaliaram-se em cada grupo 28 variedades. $\left({ }^{2}\right)$ Porcentagens médias calculadas em relação ao controle considerado como $100 \%$. Médias seguidas pelas mesmas letras, na coluna, não diferem entre si pelo teste de Tukey, ao nível de 5\%.

Para o caráter sobrevivência, constata-se no quadro 5 que os grupos japonês, filipino e brasileiro, até as doses de 60 Gy, 240 Gy e 180 Gy, respectivamente, não diferiram em relação ao controle.

$\mathrm{Na}$ figura 1, observa-se como os grupos foram afetados pela radiação gama em cada caráter. De modo geral, pode-se dizer que os grupos apresentam diferenças, entretanto, não são marcantes. Por isso, julgou-se necessário que a comparação entre grupos fosse feita por dose para que os três grupos pudessem ser especificados.

A comparação de médias de doses entre os grupos japonês, filipino e brasileiro pelo teste de Tukey para emergência, altura e sobrevivência apresenta-se no quadro 6. Em termos gerais, os três caracteres analisados nas respectivas dosagens de raios gama não apresentaram grandes diferenças. 

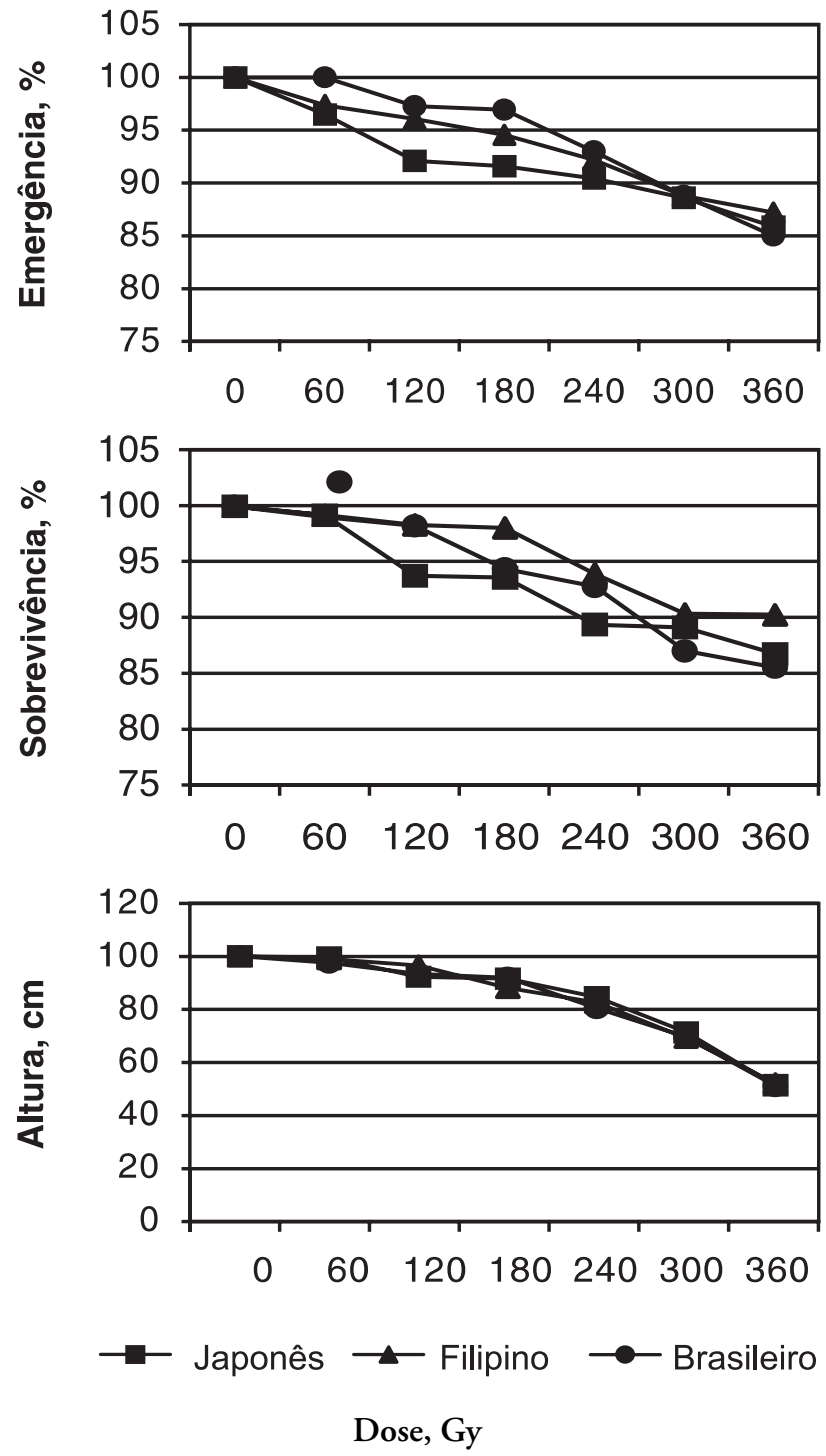

Figura 1. Relação entre doses de radiação gama e porcentagem média de emergência (A), de sobrevivência (B) e altura média de plântulas $\mathrm{M}_{1}$ em relação ao controle considerado como 100\%.

Para a emergência, o grupo filipino mostrou-se diferente do japonês nas doses de 60 Gy, 120 Gy, 180 Gy, 240 Gy e 360 Gy. O grupo brasileiro, por sua vez, apresenta comportamento semelhante aos grupos japonês e filipino, sendo igual ao japonês em todas as doses, exceto na de $120 \mathrm{~Gy}$, e igual ao filipino nas demais, exceto em $180 \mathrm{~Gy}$. $\mathrm{Na}$ dose $360 \mathrm{~Gy}$, as reduçōes da emergência foram $85,85 \%$ para o grupo japonês, $87,15 \%$ para o filipino e $85,22 \%$ para o brasileiro.

Para o caráter altura, constatou-se que os grupos não apresentaram diferenças entre si, exceto na dose de 360 Gy em que o grupo japonês difere do filipino, mas não do brasileiro na mesma dose. Na dose $360 \mathrm{~Gy}$, as reduçōes de altura foram $57,25 \%$ para o grupo japonês, $51,82 \%$ para o grupo filipino e $50,76 \%$ para o grupo brasileiro.
Quanto à sobrevivência, verificou-se que os grupos filipinos e brasileiros são iguais em todas as dosagens, exceto na dose 360 Gy. O grupo japonês, por sua vez, não difere do grupo filipino nas doses 0 e $300 \mathrm{~Gy}$ e, do grupo brasileiro, nas doses $0,180,300$ e $360 \mathrm{~Gy}$. Na dose $360 \mathrm{~Gy}$, as reduçōes no número de plântulas sobreviventes dos três grupos em relação ao controle foram $86,78 \%$ para o japonês, $88,23 \%$ para o filipino e $85,53 \%$ para o brasileiro.

É importante ressaltar que, entre os caracteres avaliados, o caráter altura foi o mais afetado pela radiação e também o que menos contribuiu para as diferenças gerais entre os grupos, ao passo que o caráter sobrevivência foi o menos afetado.

Os resultados apresentados em relação às reduções de altura e sobrevivência não estão de acordo com os encontrados, respectivamente, por FujII (1962) e Sii e

Quadro 4. Médias e porcentagens médias de altura de plântulas $M_{1}$ dentro de grupos ${ }^{(1)}$ em função das doses de radiação gama aplicadas em variedades de arroz-desequeiro. Piracicaba (SP), 1991

\begin{tabular}{|c|c|c|}
\hline Dose/Gy & Altura & Média $^{(2)}$ \\
\hline & $\begin{array}{c}\mathrm{cm} \\
\text { Japonês }\end{array}$ & $\%$ \\
\hline 0 & $13,03 \mathrm{a}$ & $(100,00)$ \\
\hline 60 & $13,07 \mathrm{a}$ & $(100,31)$ \\
\hline 120 & $12,07 \mathrm{~b}$ & $(92,63)$ \\
\hline 180 & $11,94 \mathrm{~b}$ & $(91,63)$ \\
\hline 240 & $11,04 \mathrm{c}$ & $(84,73)$ \\
\hline 300 & $9,30 \mathrm{~d}$ & $(71,37)$ \\
\hline 360 & $7,46 \mathrm{e}$ & $(57,25)$ \\
\hline CV (\%) & 16,21 & - \\
\hline \multirow[t]{2}{*}{ D.M.S. } & 0,81 & - \\
\hline & Filipino & \\
\hline 0 & $12,89 \mathrm{a}$ & $(100,00)$ \\
\hline 60 & $12,75 \mathrm{a}$ & $(98,91)$ \\
\hline 120 & $12,42 \mathrm{a}$ & $(96,35)$ \\
\hline 180 & $11,34 \mathrm{~b}$ & $(87,98)$ \\
\hline 240 & $10,64 \mathrm{~b}$ & $(82,54)$ \\
\hline 300 & $8,89 \mathrm{c}$ & $(68,97)$ \\
\hline 360 & $6,68 \mathrm{~d}$ & $(51,82)$ \\
\hline CV (\%) & 16,76 & - \\
\hline \multirow[t]{2}{*}{ D.M.S. } & 0,69 & - \\
\hline & Brasileiro & \\
\hline 0 & $13,12 \mathrm{a}$ & $(100,00)$ \\
\hline 60 & $12,79 \mathrm{ab}$ & $(97,48)$ \\
\hline 120 & $12,24 \mathrm{bc}$ & $(93,29)$ \\
\hline 180 & $12,08 \mathrm{c}$ & $(92,07)$ \\
\hline 240 & $10,52 \mathrm{~d}$ & $(80,18)$ \\
\hline 300 & $9,10 \mathrm{e}$ & $(69,36)$ \\
\hline 360 & $6,66 \mathrm{f}$ & $(50,76)$ \\
\hline CV (\%) & 15,34 & - \\
\hline D.M.S & 0,70 & - \\
\hline
\end{tabular}

(1) Avaliaram-se em cada grupo 28 variedades. $\left({ }^{2}\right)$ Porcentagens médias calculadas em relação ao controle considerado como $100 \%$. Médias seguidas pelas mesmas letras, na coluna, não diferem entre si pelo teste de Tukey, ao nível de 5\%. 
Quadro 5. Médias e porcentagens médias de sonrevivência de plântulas $M_{1}$ dentro de grupos ${ }^{(1)}$ em função das doses de radiação gama aplicadas em variedades de arroz-desequeiro. Piracicaba (SP), 1991

\begin{tabular}{|c|c|c|}
\hline Dose/Gy & Sobrevivência & Média $^{(2)}$ \\
\hline & & $\%$ \\
\hline \multicolumn{3}{|c|}{ Japonês } \\
\hline 0 & 67,99 a & $(100,00)$ \\
\hline 60 & $67,63 \mathrm{ab}$ & $(99,47)$ \\
\hline 120 & $67,42 \mathrm{~b}$ & $(99,16)$ \\
\hline 180 & $63,77 \mathrm{c}$ & $(93,79)$ \\
\hline 240 & $60,77 \mathrm{~d}$ & $(89,38)$ \\
\hline 300 & $60,61 \mathrm{~d}$ & $(89,15)$ \\
\hline 360 & $59,00 \mathrm{e}$ & $(86,78)$ \\
\hline CV (\%) & 0,40 & - \\
\hline D.M.S. & 18,00 & - \\
\hline \multicolumn{3}{|c|}{ Filipino } \\
\hline 0 & $71,44 \mathrm{a}$ & $(100,00)$ \\
\hline 60 & $70,88 \mathrm{a}$ & $(99,23)$ \\
\hline 120 & $70,23 \mathrm{a}$ & $(98,31)$ \\
\hline 180 & $70,05 \mathrm{a}$ & $(98,05)$ \\
\hline 240 & $67,15 \mathrm{ab}$ & $(93,99)$ \\
\hline 300 & $64,54 \mathrm{~b}$ & $(90,34)$ \\
\hline 360 & $63,03 \mathrm{~b}$ & $(88,23)$ \\
\hline CV (\%) & 12,10 & - \\
\hline D.M.S. & 6,80 & - \\
\hline \multicolumn{3}{|c|}{ Brasileiro } \\
\hline 0 & $71,09 \mathrm{a}$ & $(100,00)$ \\
\hline 60 & $70,40 \mathrm{~b}$ & $(99,03)$ \\
\hline 120 & $69,82 \mathrm{ab}$ & $(98,21)$ \\
\hline 180 & $67,07 \mathrm{ab}$ & $(94,35)$ \\
\hline 240 & $65,96 \mathrm{bc}$ & $(92,78)$ \\
\hline 300 & $61,84 \mathrm{c}$ & $(86,99)$ \\
\hline 360 & $60,80 \mathrm{cl}$ & $85,53)$ \\
\hline CV (\%) & 5,8 & - \\
\hline D.M.S & 19,00 & - \\
\hline
\end{tabular}

$\left.{ }^{(}\right)$Avaliaram-se em cada grupo 28 variedades. $\left({ }^{2}\right)$ Porcentagens médias calculadas em relação ao controle considerado como $100 \%$. Médias seguidas pelas mesmas letras, na coluna, não diferem entre si pelo teste de Tukey, ao nível de 5\%.

SiddiQ e Aminathan (1968). Esses autores constataram que as variedades do grupo Japônica são mais sensíveis à radiação que as variedades do grupo Índica. Entretanto, deve-se ressaltar que os autores citados utilizaram poucas variedades na comparação de radiossensitividade entre Índica e Japônica. Supōe-se que há grande variação para a radiossensitividade nesses grupos.

Conger (1976) ressaltou que a ausência ou a baixa concentração de enzimas reparadoras, catalases e peroxidases específicas na decomposição de produtos $\mathrm{da}$ radiação também podem provocar aumento da radiossensitividade. $\mathrm{O}$ fato de os grupos Índica $\mathrm{e}$ Japônica apresentarem semelhanças em relação à radiossensitividade sugere que esses grupos não
Quadro 6. Médias de emergência, altura e sobrevivência de plântulas $M_{1}$ entre grupos em função das doses de radiação gama aplicadas em arroz-de-sequeiro. Piracicaba (SP), 1991

\begin{tabular}{|c|c|c|c|c|c|}
\hline \multirow{2}{*}{ Dose } & \multicolumn{3}{|c|}{ Grupos $^{(1)}$} & \multirow{2}{*}{$\mathrm{CV} \%$} & \multirow{2}{*}{ D.M.S } \\
\hline & Japonês & Filipino & Brasileiro & & \\
\hline \multicolumn{6}{|c|}{ Emergência, \% } \\
\hline 0 & $70,61 \mathrm{~A}$ & $74,61 \mathrm{~A}$ & $71,70 \mathrm{~A}$ & 15,34 & 4,34 \\
\hline 60 & $68,13 \mathrm{~B}$ & $72,63 \mathrm{~A}$ & $71,89 \mathrm{AB}$ & 15,67 & 4,26 \\
\hline 120 & $64,67 \mathrm{~B}$ & $70,53 \mathrm{~A}$ & $69,97 \mathrm{~A}$ & 15,06 & 4,08 \\
\hline 180 & $65,03 \mathrm{~B}$ & $71,66 \mathrm{~A}$ & 66,67 B & 16,48 & 4,45 \\
\hline 240 & $62,55 \mathrm{~B}$ & $68,76 \mathrm{~A}$ & $66,43 \mathrm{AB}$ & 17,01 & 4,41 \\
\hline 300 & $63,82 \mathrm{~A}$ & $66,24 \mathrm{~A}$ & $63,81 \mathrm{~A}$ & 18,74 & 4,48 \\
\hline 360 & $60,62 \mathrm{~B}$ & $65,02 \mathrm{~A}$ & $61,10 \mathrm{AB}$ & 18,47 & 4,30 \\
\hline \multicolumn{6}{|c|}{ Altura, $\mathrm{cm}$} \\
\hline 0 & $13,03 \mathrm{~A}$ & $12,89 \mathrm{~A}$ & $13,12 \mathrm{~A}$ & 20,07 & 1,03 \\
\hline 60 & $13,07 \mathrm{~A}$ & $12,75 \mathrm{~A}$ & $12,79 \mathrm{AB}$ & 19,84 & 1,02 \\
\hline 120 & $12,07 \mathrm{~A}$ & $12,42 \mathrm{~A}$ & $12,24 \mathrm{~A}$ & 20,15 & 0,99 \\
\hline 180 & $11,94 \mathrm{~A}$ & $11,34 \mathrm{~A}$ & $12,08 \mathrm{~A}$ & 20,10 & 0,95 \\
\hline 240 & $11,04 \mathrm{~A}$ & $10,64 \mathrm{~A}$ & $10,52 \mathrm{~A}$ & 21,91 & 0,94 \\
\hline 300 & $9,30 \mathrm{~A}$ & $8,89 \mathrm{~A}$ & $9,10 \mathrm{~A}$ & 23,12 & 0,86 \\
\hline 360 & $6,68 \mathrm{~B}$ & $7,46 \mathrm{~A}$ & $6,66 \mathrm{~B}$ & 29,14 & 0,77 \\
\hline \multicolumn{6}{|c|}{ Sobrevivência, \% } \\
\hline 0 & $67,99 \mathrm{~A}$ & $71,44 \mathrm{~A}$ & $71,09 \mathrm{~A}$ & 15,61 & 3,50 \\
\hline 60 & $67,63 \mathrm{~B}$ & $70,88 \mathrm{~A}$ & $70,40 \mathrm{~A}$ & 15,98 & 2,70 \\
\hline 120 & $67,42 \mathrm{~B}$ & $70,23 \mathrm{~A}$ & $69,82 \mathrm{~A}$ & 16,18 & 2,30 \\
\hline 180 & $63,77 \mathrm{~B}$ & $70,05 \mathrm{~A}$ & $67,07 \mathrm{AB}$ & 16,81 & 3,50 \\
\hline 240 & $60,77 \mathrm{~B}$ & $67,15 \mathrm{~A}$ & $65,96 \mathrm{~A}$ & 20,19 & 2,90 \\
\hline 300 & $60,61 \mathrm{~A}$ & $64,54 \mathrm{~A}$ & $61,84 \mathrm{~A}$ & 20,60 & 3,95 \\
\hline 360 & $59,00 \mathrm{~B}$ & $64,03 \mathrm{~A}$ & $60,80 \mathrm{~B}$ & 24,29 & 2,00 \\
\hline
\end{tabular}

(') Avaliaram-se em cada grupo 28 variedades. Médias seguidas pelas mesmas letras, na linha, não diferem entre si pelo teste de Tukey, ao nível de 5\%.

apresentam alelos específicos que os diferenciam na produção de enzimas reparadoras, catalases e peroxidases, o que se explica pela origem dos grupos.

O grupo Índica e Japônica tiveram origem a partir de um ancestral comum (ОKA, 1958, 1988; MORISHIMA е ОКА, 1981). Desse modo, entre eles existem semelhanças genéticas, principalmente para várias isoenzimas.

Supõe-se que a maioria das variedades brasileiras utilizadas neste estudo, pertença ao grupo Japônica (Rangel) $)^{(4)}$. Esse fato poderá demonstrar, ainda mais, a semelhança verificada entre os grupos Índica e Japônica em relação à radiossensitividade, pois era esperado que o grupo das variedades brasileiras não apresentasse diferenças significativas em relação ao grupo das variedades japonesas para a maioria das doses em todos os caracteres avaliados. Entretanto, constatou-se que as variedades brasileiras têm comportamento similar para sensitividade à radiação gama em relação aos grupos filipino e japonês, ou seja, ora é semelhante ao grupo filipino, ora ao japonês, dependendo da dosagem utilizada. 


\section{CONCLUSÕES}

1. Dentro e entre grupos de variedades procedentes do Japão, Filipinas e Brasil, houve diferenças de radiossensitividade para todos os caracteres avaliados.

2. A altura foi o caráter mais afetado pela radiação e o que menos contribuiu para as diferenças gerais entre os grupos.

3. O caráter sobrevivência foi o menos afetado pela radiação.

\section{AGRADECIMENTOS}

À Coordenadoria de Aperfeiçoamento de Pessoal de Ensino Superior (CAPES) pelo apoio financeiro.

\section{REFERÊNCIAS BIBLIOGRÁFICAS}

CALDECOTT, R.S. Effects of hydration on X-ray sensitivity in Hordeum. Radiation Research, San Diego, v.3, p.316330, 1955.

CONGER, B.V. Response of inbred and hybrid maize seed to gamma radiation and fission neutrons and its relationship to nuclear volume. Environmental and Experimental Botany, London, v.16, p.165-170, 1976.

FERREIRA, M.E.; PENTEDO, M.I.O; BRONDANI, C.; FERREIRA, M.A.; RANGEL, P.H.N. Caracterización y uso de marcadores RAPD y microsatélites (SSR) en el monitoreo del programa de mejoramiento poblacional en arroz. In: GUIMARÃES, E.P. Avances en el mejoramiento poblacional en arroz. Santo Antônio de Goiás: Embrapa Arroz e Feijão, 2000. p.37-62.

FUJII, T. Radiosensitivity in plants: V. Experiments with several cultivated and wild rices. Japanese Journal Breeding, Tokyo, v.12, p.131-136, 1962.

GAUL, H. Plant injury and lethality. In: INTERNATIONAL ATOMIC ENERGY AGENCY. Manual on mutation breeding. 2.ed. Vienna, 1977. p.87-91. (Technical reports series, 119).

GUIMARÃES, E.P.; BORRERO, J.; OSPINA-REY, Y. Genetic diversity of upland rice germplasm distributed in Latin America. Pesquisa Agropecuária Brasileira, Brasília, v.31, n.3, p.187-194, 1996.
JOHNSON, E.L. The influence of X-radiation in Atriplex hortensis L. New Phytologist, London, v.32, p.297-307, 1933.

KATO, S.; KOSAKA H.; HARA, S. On the affinity of rice varieties as show by fertility of hybrid plants. Bulletin Scientific Faculty Agricultural Kyushu University, Fukuoka, v.3, p. 132-147, 1928.

KHUSH, G.S. Selecting rice for simply inherited resistance. In: SYMPOSIUM ON PLANT BREEDING IN THE 1990s, 1991, Raleigh. Proceedings... STAALKER, H.T.; MURPHY, J.P. (Eds.). Wallingford: CAB, 1992. cap.13, p.303-322.

MONTALVÁN, R.; DESTRO, D.; SILVA, E.F.; MONTAÑO, D.C. Genetic base of Brazilian upland rice cultivars. Journal of Genetics \& Breeding, Rome, v.53, n.3, p.203-209, 1998.

MORISHIMA, H.; OKA, H.I. Phylogenetic differentiation of cultivated rice. Japanese Journal Breeding, Tokyo, v.31, p.402-413, 1981.

OKA, H.I. Indica-japonica differentiation of rice cultivars. In: OKA, H.I. Origin of cultivated rice. Amsterdan: Elsevier, 1988. p.141-170.

OKA, H.I. Intervarietal variation and classification of cultivated rice. Indian Journal of Genetics \& Plant Breeding, New Delhi, v.18, p.79-89, 1958.

ROBERTS, E.H. An investigation of inter varietal differences in dormancy and viability of rice seed. Annals of Botany, London, v.27, p.365-367, 1963.

SARIC, M. The effects of irradiation in relation to the biological traits of seed irradiated. In: INTERNATIONAL ATOMIC ENERGY AGENCY. Effects of ionizing radiation on seeds. Vienna, 1961. p.103-115.

SIDDIQ, E.A.; SWAMINATHAN, M.S. Induced mutations in relation to the breeding and phylogenetic differentiation of Oryza sativa. In: INTERNATIONAL ATOMICENERGY AGENCY. Rice breeding with induced mutations. Vienna, 1968. p.25-33. (Technical reports series, 86).

SMITH, L. Hereditary susceptibility to X-ray injury in Triticum Monococcum. American Journal Botany, London, v.24, p.189-191, 1942. 